\title{
Ocurrencia de Enteroparásitos en Poblaciones de Quiscalus lugubris (Aves: Passeriformes, Icteridae) del Semiárido Urbano del Estado Falcón, Venezuela
}

\author{
Occurrence of Enteric Parasites in Populations of Quiscalus lugubris (Aves: \\ Passeriformes, Icteridae) of Urban Semiarid of Falcon State, Venezuela
}

\author{
Dalmiro Cazorla Perfetti ${ }^{1,2}$, Pedro Morales Moreno ${ }^{1}$
}

\section{Resumen}

Los parásitos intestinales patógenos de aves paseriformes pueden potencialmente tener importancia zoonótica. Se realizó un estudio para determinar la ocurrencia de parásitos intestinales en heces de poblaciones de Quiscalus lugubris («torditos negros») (Aves, Passeriformes: Icteridae) en la ciudad de Coro, estado Falcón, Venezuela. El diagnóstico parasitológico de las muestras $(\mathrm{n}=156)$ se hizo mediante los métodos directo, flotación de Willis-Molloy y Faust, sedimentación en tubo y coloración de Kinyoun. Se detectaron hasta tres taxones de protozoarios, helmintos y acantocéfalos en 109 muestras $(69.9 \%)$. Isospora spp (59.0\%), Criptosporidium spp (37.8\%) y el acantocéfalo Mediorhynchus spp (6.4\%) fueron los enteroparásitos más frecuentes. Todos los parásitos encontrados representan nuevos registros para $Q$. lugubris en Venezuela.

Palabras clave: Quiscalus lugubris, parásitos intestinales, ocurrencia, Venezuela

\section{Abstract}

Intestinal parasites in passerine birds can potentially have zoonotic importance. A study was conducted to determine the occurrence of intestinal parasites in feces of Carib Grackle, Quiscalus lugubris, populations (Aves, Passeriformes: Icteridae), in the city of Coro, Falcon State, Venezuela. Parasitological examination of the samples $(n=156)$ was

\footnotetext{
${ }^{1}$ Laboratorio de Entomología, Parasitología y Medicina Tropical (L.E.P.A.M.E.T.), Centro de Investigaciones Biomédicas (C.I.B.), Decanato de Investigaciones, Universidad Nacional Experimental «Francisco de Miranda»(UNEFM), Estado Falcón, Venezuela

2E-mail: lutzomyia@hotmail.com / cdalmiro@gmail.com
}

Recibido: 28 de junio de 2016

Aceptado para publicación: 29 de octubre de 2016 
conducted by applying the direct smear, Willis-Molloy $(\mathrm{NaCl})$ and Faust (zinc sulphate) flotation methods, spontaneous sedimentation in tube technique and Kinyoun staining. One or up to three intestinal protozoa, helminths and acanthocephalan taxa were detected in $109(69.9 \%)$ fecal samples. Isospora spp (59.0\%), Criptosporidium spp (37.8\%) and the spiny-headed worm Mediorhynchus spp (6.4\%) were the most frequent enteric parasites. All of the detected parasites are new records for $Q$. lugubris in Venezuela.

Key words: Quiscalus lugubris, intestinal parasites, occurrence, Venezuela

\section{INTRODUCCIÓN}

Quiscalus es un género de aves paseriformes americanas de la familia Icteridae que se encuentra integrado por siete especies y una amplia variedad de subespecies (Powell et al., 2008). Para Venezuela, se han reportado las especies $Q$. mexicanus y $Q$. lugubris. La primera de estas se le denomina «galandra» (great-tailed grackle), y solo ha sido capturada en el estado Zulia, región noroccidental, en simpatría con Q. lugubris (Phelps y Meyer de Shauensee, 1994; Casler y Esté, 1996; Hilty et al., 2003). Por contraste, Q. lugubris, denominada comúnmente como «tordo»o «tordito negro», posee una distribución más amplia, especialmente hacia la región septentrional del territorio nacional, siendo reportadas dos subespecies (Q. lugubris lugubris y $Q$. lugubris insularis). Esta especie se le encuentra distribuida desde las Antillas Menores, Trinidad y Tobago hasta el norte de Suramérica (Colombia, Brasil y las Guayanas), donde se le conoce como «zanate caribeño» o «chango llanero» («carib grackle») (Phelps y Meyer de Shauensee, 1994; Casler y Esté, 1996; Hilty et al., 2003; Sanz et al., 2010).

Q. lugubris, al igual que Q. mexicanus, es un taxón de ave muy gregaria que se encuentra bien adaptada a los ambientes modificados o intervenidos por el hombre, por lo que es habitual visualizar sus poblaciones alrededor de muchos sitios de explotación animal (e.g., fincas de ganado vacuno, granjas avícolas, cochineras) y de cultivos agrícolas, así como también dentro de las áreas urbanizadas (e.g., plazas, mercados, restaurantes). En estos lugares, al ser aves oportunistas y omnívoras, encuentran una fuente asequible y continua de alimento a partir de los desechos (Strewe et al., 2006; Minott y Caballero, 2007).

Esta afinidad hacia los ambientes sinantrópicos las convierte en potenciales portadores de agentes infecciosos (entéricos, tisulares), tanto de interés médico-zoonótico como veterinario, lo cual se podría potenciar por la afinidad de estas aves por los reservorios de agua y su conocida conducta alimentaria de mojado («dunking») (MorandFerron et al., 2006; Minott y Caballero, 2007). Sin embargo, es muy poco lo que se ha estudiado acerca de la presencia de patógenos, incluyendo parásitos intestinales, en las poblaciones de Q. lugubris. Esto contrasta con las investigaciones hechas en otras especies de aves, ya sea de este o de otros géneros de paserinos, donde se han reportado virus como el de la influenza aviar (A/H7N3), del Nilo Occidental, de la encefalitis de San Luis y de la enfermedad Newcastle; bacterias como Chlamydophila psittaci, Salmonella spp, E. coli O157:H7 y Yersinia enterocolitica; hongos (Histoplasma capsulatum) y protozoarios coccidios tisulares como Toxoplasma gondii, Sarcocystis spp o intestinales del género Cryptosporidum spp (Shayegani et al., 1986; Andersen y Vanrompay, 2003; Minott y Caballero, 2007; Alvarado-Esquivel et al., 2011; Sevá et al. 2011; Callaway et al., 2014; Navarro-Lopez et al., 2014). 
En la ciudad de Coro, enclavada en la zona semiárida del estado Falcón, región noroccidental de Venezuela, este equipo de investigadores ha venido observando los sitios donde pernoctan y se alimentan las poblaciones de aves paseriformes, incluyendo las cercanías de los mercados, expendios de comidas (restaurantes), viviendas y depósitos de basura. Es por ello que, ante su potencial de transmisores de enfermedades zoonóticas, se investigó la ocurrencia de parásitos gastrointestinales en muestras fecales de poblaciones de $Q$. lugubris en la ciudad de Coro.

\section{Materiales y Métodos}

El estudio se llevó a cabo entre noviembre y diciembre de 2015, en la ciudad de Coro, municipio Miranda, estado Falcón, Venezuela. La región posee una zona bioclimática del tipo Monte Espinoso Tropical (MET), cuyas características han sido reseñadas en una publicación previa (Cazorla y Morales, 2013).

Previo a la investigación, se observaron los sitios donde pernoctaban las poblaciones de $Q$. lugubris, y cuya identificación taxonómica se hizo por morfología comparativa utilizando las ilustraciones de Phelps y Meyer de Schauensee (1994), ya sea visualizándolas con binoculares (Figura 1b) o con ejemplares hallados muertos (Figura 1a). Dentro de estos lugares, se optó por tomar muestras fecales en la base de dos árboles muy frondosos de la familia de las leguminosas (Figura 1b), considerando las decenas de ejemplares de «torditos negros» que integran a estas poblaciones, así como por su ubicación frente a restaurantes céntricos muy concurridos. La distancia entre ambos árboles era de $20 \mathrm{~m}$. Además, se visualizaba la abundante contaminación fecal de las áreas adyacentes (Figuras 1c, 1d). Es significativo señalar que estas aves son muy territoriales, no permitiendo la presencia de otras aves en sus sitios de pernocta (Phelps y Meyer de Shauensee, 1994; Casler y Esté, 1996; Hilty et al., 2003).
Durante un mes y a primeras horas de la mañana (06:30-07:00), se recolectaron diariamente entre 5-6 muestras de heces frescas de Q. lugubris en puntos equidistantes de 2.5 a $3 \mathrm{~m}$. En total, se colectaron 156 muestras en envases estériles herméticos, que fueron llevadas en cavas de poliuretano al Laboratorio de Entomología, Parasitología y Medicina Tropical (LEPAMET) del Centro de Investigaciones Biomédicas (CIB), Universidad Nacional Experimental Francisco de Miranda (UNEFM). Las muestras fecales recogidas eran características de Q. lugubris, pues presentan una porción blanquecina y otra de coloración marrón (Figuras 1c, 1d). Las muestras fueron analizadas el día de la toma, caso contrario, se preservaron y fijaron, añadiéndoles $1-2 \mathrm{ml}$ de formaldehído al $10 \%$.

Las muestras fecales se procesaron por el método coprológico directo y por las técnicas de flotación simple de Willis-Molloy con solución sobresaturada de cloruro de sodio $(\mathrm{NaCl})$, flotación-centrifugación de Faust con sulfato de zinc $\left(\mathrm{ZnSO}_{4}\right)$, de sedimentación en tubo (TSET) y coloración de Kinyoun (ZiehlNeelsen modificado) para la detección de los ooquistes ácido resistentes de coccidios intestinales (e.g. Cryptosporidium spp) (Morales y Pino de Morales, 1977; Botero y Restrepo, 2012). Los ooquistes de coccidios fueron medidos empleando el micrómetro ocular. Las heces fueron observadas y fotografiadas (Olympus, Fe-120, Olympus Imaging Corp, Japón) por duplicado bajo microscopio de luz (Axiostar Plus, Carl Zeiss, Alemania) con y sin solución yodada de lugol.

La identificación de las taxa parasitarias se realizó mediante morfología externa e interna comparada de quistes-ooquistes/ trofozoitos y huevos/larvas (Moore 1962, Kaufmann, 1996, Norton y Ruff, 2003; Botero y Restrepo, 2012). Las heces se cultivaron 2-5 días en una solución de dicromato de potasio al $2.5 \%$ a temperatura ambiental para diferenciar coccidios de los géneros Eimeria e Isospora, en caso de que no se observasen ooquistes esporulados (Restrepo y Carvajal, 1982). 

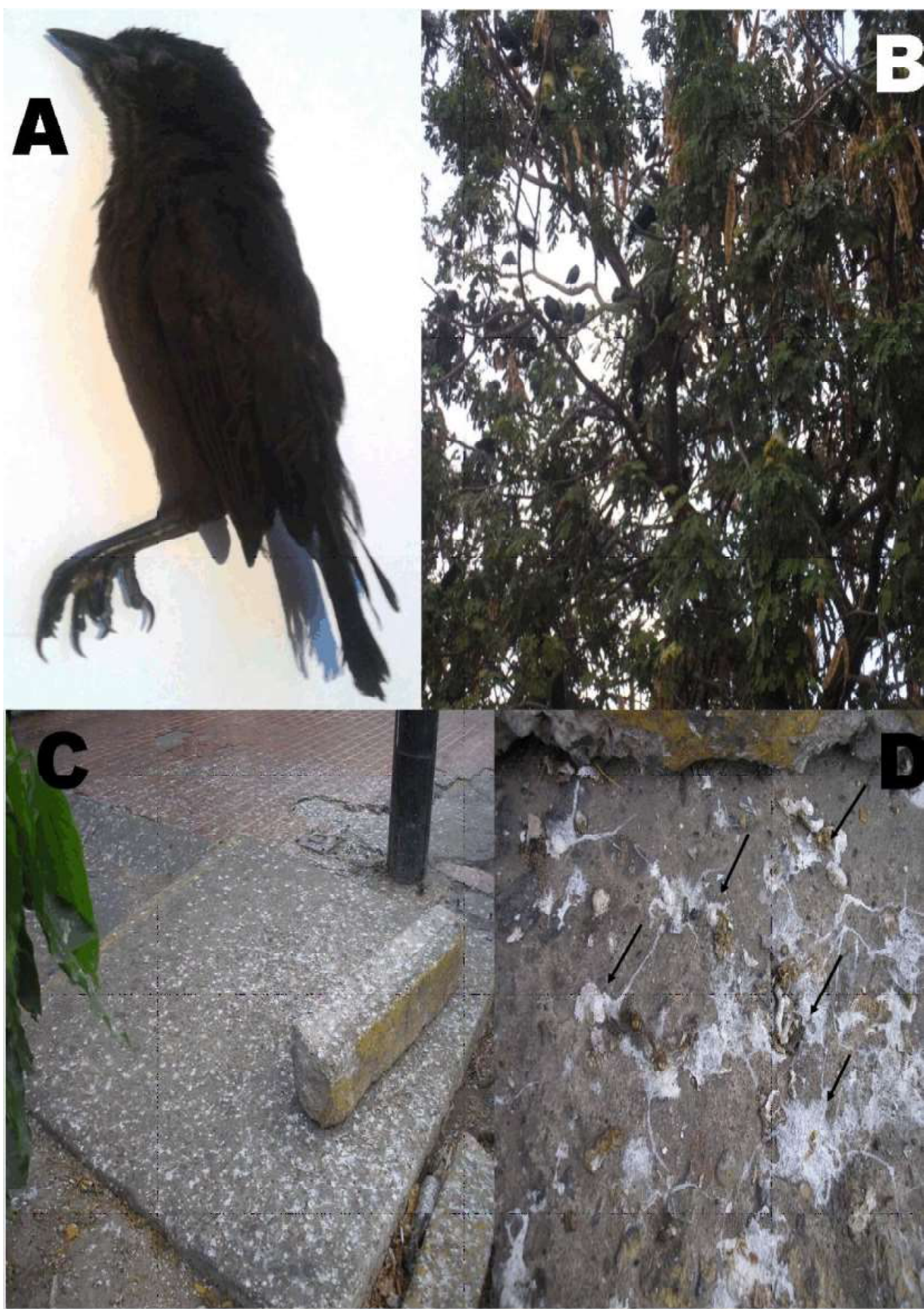

Figura 1. (a) Ejemplar muerto de Quiscalus lugubris. (b). Población de Q. lugubris en árbol de leguminosa cerca de locales de restaurantes. (c) Superficie de cemento (acera) en la base de los árboles donde se observan las deyecciones de las poblaciones de $Q$. lugubris. (d) Se detalla las características de las deyecciones (flechas) de Q. lugubris 
Las muestras se consideraron positivas a parásitos cuando se detectaba al menos un estadio (quiste/ooquistes/huevo/larva) de protozoario o helminto. Los porcentajes de positividad se expresaron como ocurrencia en lugar de prevalencia (además del intervalo de confianza de1 95\%), debido a que varias de las muestras fecales pudieron haberse originado de la misma ave (Rózsa et al., 2000).

\section{Resultados y Discusión}

La ocurrencia global de muestras con parásitos intestinales fue de $69.9 \%$ (IC: 62.2 - 77.8\%) (109/156). La ocurrencia de los taxones de protozoarios, helmintos y acantocéfalos se muestra en el Cuadro 1. Dentro de los protozoarios, solo se detectaron los apicomplejos coccidios de los géneros Isospora spp (59.0\%) y Criptosporidium spp (37.8\%), en tanto que solo se observaron huevos con morfología compatible al género Mediorhynchus (Gigantorhynchida: Gigantorhynchidae), entre los acantocéfalos, con 6.4\% (Cuadro 1, Figura 2).

El monoparasitismo se presentó en el $30.1 \%(47 / 109)$ de las muestras positivas a parásitos gastrointestinales, mientras que en el caso de las infecciones múltiples se detectaron muestras hasta con tres taxones de parásitos (Cuadro 2).

El estudio de los microorganismos, incluyendo los intestinales, ya sean hongos, virus, bacterias, protozoos o helmintos en las poblaciones de aves silvestres, es de especial interés para la salud pública, debido a la presencia de agentes de importancia zoonótica. En el caso particular de $Q$. lugubris, cuyas poblaciones se encuentran bien adaptadas a los ambientes modificados por el humano, el presente estudio es de gran relevancia, ya que estas aves paserinas podrían servir como vehículo de estos agentes infecciosos para el humano y sus animales a través de la contaminación ambiental. Esta misma consideración es necesaria tener en cuenta para las aves que se mantienen en cautiverio, las cuales son más susceptibles a las infecciones debido a la alteración de sus sistemas inmunes (Cushing et al., 2011).

Isospora spp fue el parásito entérico de mayor ocurrencia en las muestras fecales analizadas de $Q$. lugubris. Hasta donde se ha podido indagar, este es el primer registro de Isospora spp en Q. lugubris y el segundo para aves silvestres en el territorio nacional (I. tiaris en Tiaris fuliginosa, Passeriformes-Thraupidae) (Ball y Daszak, 1997), y el tercero para el género Quiscalus (Boughton et al., 1938; Minott y Caballero, 2007). La infección por Isospora spp en paserinos puede instaurarse a nivel intestinal y a nivel sistémico o extraintestinal, también denominada como atoxoplasmosis, la cual, por lo general, coexiste de manera endémica con las aves silvestres; sin embargo, cuando estas se mantienen en cautiverio, la infección tiende a ser fulminante y fatal debido al estrés, el hacinamiento, la inmunosupresión y las infecciones concomitantes. Inclusive, se tiene evidencia que la infección sistémica puede inducir una transformación neoplásica (linfomas) (Gill y Paperna, 2008; Cushing et al. 2011). En este mismo sentido, la urbanización y la presencia humana se encuentran asociadas al incremento de las infecciones enteroparasitarias y particularmente de Isospora spp en aves Passeriformes (Giraudeau et al., 2014).

Se debe resaltar el hallazgo de ooquistes de Cryptosporidum spp en el $37.8 \%$ de las muestras en el presente estudio. Este parece ser el primer reporte de este coccidio en el género Quiscalus y por extensión en «torditos negros», el quinto en la familia Icteridae, compuesta por 95 especies en 23 géneros (Chelladurai et al., 2016), y el segundo para Venezuela en aves silvestres (Díaz-Ungría, 1981; Arcay y Bruzual, 1993); lo que pone en evidencia, según lo indican Chelladurai et al. (2016), que la mayoría de estudios sobre este coccidio apicomplejo se han realizado en aves cautivas. 
Cuadro 1. Frecuencia absoluta (N) y ocurrencia (\%) de parásitos intestinales en Quiscalus lugubris, en Coro, estado Falcón, Venezuela (2015)

\begin{tabular}{lccc}
\hline & & \multicolumn{2}{c}{ Muestras positivas } \\
\cline { 3 - 4 } Taxón & $\begin{array}{c}\text { Muestras } \\
(\mathrm{n})\end{array}$ & $\begin{array}{c}\text { Frecuencia } \\
(\%)\end{array}$ & $\begin{array}{c}\text { Intervalo de } \\
\text { confianza } \\
(95 \%)\end{array}$ \\
\hline $\begin{array}{l}\text { Protozoarios } \\
\quad \text { Isospora spp }\end{array}$ & 92 & 59.0 & $51.2-66.8$ \\
$\quad \begin{array}{l}\text { Criptosporidium spp } \\
\text { Helmintos }\end{array}$ & 59 & 37.8 & $30.2-45.8$ \\
$\quad \begin{array}{l}\text { Nematodo no identificado } \\
\text { Gnathostomatídeo no identificado }\end{array}$ & 2 & & 0.6 \\
$\quad \begin{array}{l}\text { Acantocéfalos } \\
\quad \text { Mediorhynchus spp }\end{array}$ & 1 & 1.3 & $0.01-3.3$ \\
\hline Total & 10 & 6.4 & $2.5-10.3$ \\
\hline
\end{tabular}

Cuadro 2. Asociación entre parásitos intestinales en Quiscalus lugubris en Coro, estado Falcón, Venezuela (2015)

\begin{tabular}{|c|c|c|c|}
\hline \multirow[b]{2}{*}{ Taxón } & \multirow[b]{2}{*}{$\begin{array}{l}\text { Muestras } \\
\quad(\mathrm{n})\end{array}$} & \multicolumn{2}{|c|}{ Muestras positivas } \\
\hline & & $\begin{array}{l}\text { Frecuencia }^{1} \\
\quad(\%)\end{array}$ & $\begin{array}{c}\text { Intervalo de } \\
\text { confianza } \\
(95 \%)\end{array}$ \\
\hline \multicolumn{4}{|l|}{ Monoparasitados } \\
\hline Isospora spp & 35 & 31.1 & $24.2-39.8$ \\
\hline Criptosporidium spp & 11 & 10.1 & $4.1-15.9$ \\
\hline Mediorhynchus spp & 1 & 0.8 & $0.01-3.0$ \\
\hline \multicolumn{4}{|l|}{ Biparasitados } \\
\hline Isospora spp / Cryyptosporidium $\mathrm{spp}$ & 45 & 41.3 & $31.2-50.8$ \\
\hline Isospora spp / Mediorhynchus spp & 7 & 6.4 & $2.5-10.3$ \\
\hline Isospora spp /Gnathostomatídeo & 1 & 0.8 & $0.01-3.0$ \\
\hline Isospora spp / Nematodo no identificado & 1 & 0.8 & $0.01-3.0$ \\
\hline \multicolumn{4}{|l|}{ Triparasitados } \\
\hline $\begin{array}{l}\text { Isospora spp / Cryyptosporidium } \mathrm{spp} / \\
\text { Mediorhynchus } \mathrm{spp}\end{array}$ & 2 & 1.8 & $0.01-3.8$ \\
\hline $\begin{array}{l}\text { Isospora spp / Cryyptosporidium spp / } \\
\text { Gnathostomatídeo }\end{array}$ & 1 & 0.8 & $0.01-3.0$ \\
\hline
\end{tabular}




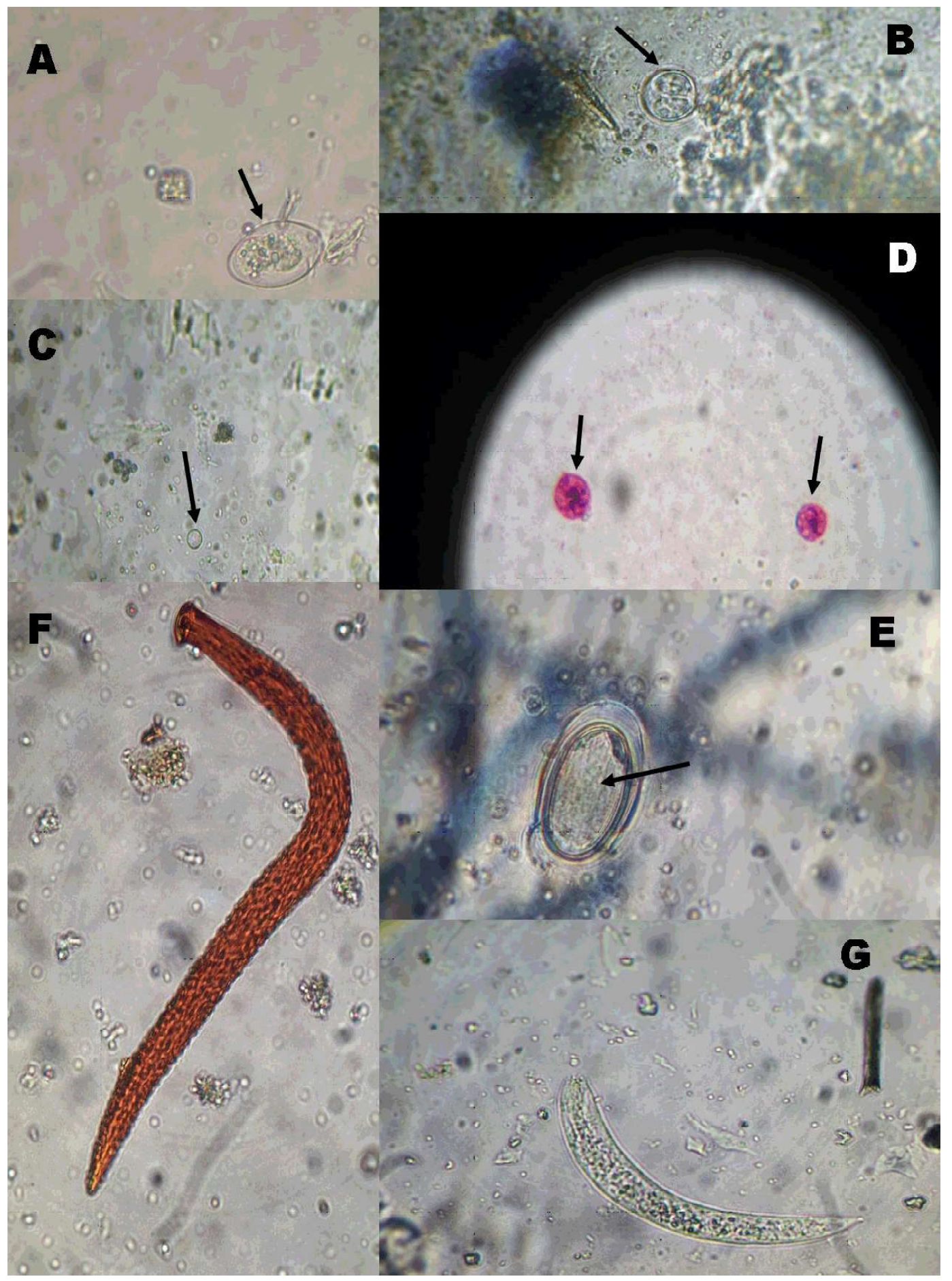

Figura 2. Taxones enteroparasitarios aislados en muestras fecales de Quiscalus lugubris en la ciudad de Coro, estado Falcón, Venezuela (2015). Ooquistes (flechas) sin esporular (a) y esporulado (b) de Isospora spp. Ooquistes (flechas) de Cryptosporidium spp en examen directo (c) y coloración de Kinyoun (d) (1000X); (e) Huevo con acantor (flecha) de acantocéfalo (Mediorhynchus sp.); f. Larva de nematodo Gnathostomatideo (tinción Lugol); (g) Larva de nematodo no identificado (400X) 
Cryptosporidium spp es un taxón de protozoarios apicomplejos, de hábitos entéricos, de amplia distribución mundial, de mucha relevancia a nivel médico y veterinario al ocasionar la criptosporidiosis, y que sorpresivamente se le ha señalado de estar más relacionado con las gregarinas que con los coccidios Cyclospora spp y Cystoisospora spp (Botero y Restrepo, 2012; Cavalier-Smith, 2014; Ryan y Hijjawi, 2015). La criptosporidiosis es una importante enteroparasitosis que afecta hasta 17 de los 26 órdenes de aves silvestres y domesticadas, incluyendo a las del orden Passeriformes, a quienes puede ocasionar desórdenes entéricos y respiratorios con elevadas tasas de mortalidad. $C$. baileyi, C. galli y C. meleagridis son las especies más frecuentemente detectadas, además de otras taxa o genotipos que aún no se han caracterizado (Xiao, 2010; Quah et al. 2011; Sevá et al., 2011, Nakamura y Meireles, 2015). En Venezuela, mediante métodos moleculares solo se ha logrado identificar cuatro especies: C. hominis, $C$. parvum, C. canis y C. ubiquitum (Blanco et al., 2015). A la luz de todo lo comentado, se hace necesario incrementar estudios epidemiológicos con técnicas moleculares para determinar con exactitud las especies de Cryptosporidium spp presentes en las poblaciones de $Q$. lugubris, así como su capacidad zoonótica.

Los huevos del acantocéfalo Mediorhynchus sp, el cual se encuentra compuesto por más de 40 especies que parasitan aves alrededor del globo terráqueo (Smales, 2000), fueron aislados en 10 (6.4\%) muestras. Este reporte sería el primero de este taxón para $Q$. lugubris en Venezuela. Todos los integrantes del filo Acanthocephala son de vida parasitaria, pero solo algunas taxa poseen interés, tanto veterinario como médico (acantocefalosis o acantacefaliasis). Su adquisición se hace principalmente por la ingestión de peces crudos (hospedadores paraténicos) o artrópodos (Haustein et al., 2010), los cuales forman parte de la dieta de los «torditos negros» (Strewe et al., 2006; Minott y Caballero, 2007; Rodríguez-Ferraro, 2015). No obstante, al Mediorhynchus spp no se le considera de importancia para la salud animal o humana.

Las larvas de un nematodo con morfología compatible con los de la familia Gnathostomatidae se detectaron en dos muestras fecales (1.3\%), sin poder identificarlas a nivel de género. El género Gnathostoma spp presenta varias especies que son frecuentemente detectadas en vertebrados, y que pueden infectar al humano mediante la ingestión de carnes crudas o semicrudas de hospedadores secundarios infectados (pescados, ranas, serpientes e incluso pollos). Las aves también pueden actuar como hospedadores paraténicos, y se les considera como «acumuladores de larvas por excelencia» (Botero y Restrepo, 2012; GarcíaMárquez et al., 2014).

La elevada ocurrencia de parásitos intestinales, muchos de ellos potencialmente de transmisibilidad zoonótica, detectada en poblaciones de «torditos negros» en la presente investigación resalta su relevancia para las comunidades expuestas, particularmente en la ciudad de Coro, poniendo en evidencia el grado de contaminación ambiental. Los resultados de este estudio dan las bases de tipo biológico a las autoridades de salud para desarrollar e implementar programas de control y manejo integrado, que incluya educación para la salud.

\section{Conclusiones}

- La ocurrencia de parásitos intestinales en poblaciones de Q. lugubris de la ciudad de Coro, estado Falcón, Venezuela fue del $69.9 \%$.

- Todas las taxa parasitarias encontradas fueron nuevos registros en Venezuela para esta especie de ave paseriforme. 


\section{Literatura Citada}

1. Alvarado-Esquivel C, Rajendran C, Ferreira L, Kwok O, Choudhary S, Alvarado-Esquivel D, RodríguezPeña S, et al. 2011. Prevalence of Toxoplasma gondii infection in wild birds in Durango, Mexico. J Parasitol 97: 809-812. doi: 10.1645/GE-2844.1

2. Andersen A, Vanrompay D. 2003. Avian chlamydiosis (psittacosis, ornithosis). In: Saif Y, Barnes H, Fadly A, et al. (eds). Diseases of poultry. $11^{\text {th }}$ ed. Ames, Iowa: Blackwell Publishing. p 863-879.

3. Arcay L, Bruzual E.1993. Cryptosporidium en ríos de Venezuela. Encuesta epidemiológica de una población humana y fauna en convivencia. Parasitol al Día 17: 11-18.

4. Ball J, Daszak P. 1997. Isospora tiaris n.sp. (Apicomplexa: Eimeriidae) from the sooty grassquit (Tiaris fuliginosa), a passeriform bird of South America. J Parasitol 83: 465-466.

5. Blanco M, de Lucio A, Fuentes I, Carmena D. 2015. Cryptosporidium ubiquitum in Venezuela: First report in a paediatric patient with acute diarrhoea. Enferm Infecc Microbiol Clin 34: 142-143. doi: 10.1016/j.eimc.2015.05.010

6. Botero D, Restrepo M. 2012. Parasitosis humana. $4^{a}$ ed. Colombia: Corporación para Investigaciones Biológicas. $733 \mathrm{p}$.

7. Boughton D, Boughton R, Volk J. 1938. Avian hosts of the genus Isospora (Coccidiida). Ohio J Sci 38: 149-163.

8. Callaway T, Edrington T, Nisbet D. 2014. Isolation of Escherichia coli O157:H7 and Salmonella from migratory brown-headed cowbirds (Molothrus ater), common Grackles (Quiscalus quiscula), and cattle egrets (Bubulcus ibis). Foodborne Pathog Dis 11: 791-794. doi: 10.1089/fpd.2014.1800
9. Casler C, Esté E. 1996. Avifauna del manglar en la Península Ana María Campos, Estrecho del Lago de Maracaibo, Venezuela. Bol Centro Invest Biol 30: 9-44.

10. Cavalier-Smith T. 2014. Gregarine siteheterogeneous 18S rDNA trees, revision of gregarine higher classification, and the evolutionary diversification of Sporozoa. Eur J Protistol 50: 472-495. doi: 10.1016/ j.ejop.2014.07.002

11. Cazorla D, Morales P. 2013. Prevalencia de parásitos intestinales en gallos de pelea de la ciudad de Coro, estado Falcón, Venezuela. Rev Inv Vet Perú 24: 489-502. doi: 10.15381/rivep.v24i4.2748

12. Chelladurai J, Clark M, Kváè M, Holubová N, Khan E, Stenger B, Giddings C, McEvoy J. 2016. Cryptosporidium galli and novel Cryptosporidium avian genotype VI in North American red-winged blackbirds (Agelaius phoeniceus). Parasitol Res 115: 1901-1906. doi: 10.1007/s00436016-4930-8

13. Cushing T, Schat K, States S, Grodio J, O'Connell P, Buckles E. 2011. Characterization of the host response in systemic isosporosis (atoxoplasmosis) in a colony of captive American goldfinches (Spinus tristis) and house sparrows (Passer domesticus). Vet Pathol 48: 985-992. doi: 10.1177/0300985810391114

14. Díaz-Ungría C.1981. Protozoos de Venezuela. Kasmera 9: 147-215.

15. García-Márquez L, León-Règagnon $V$, Lamothe-Argumedo R, OsorioSarabia D, García-Prieto L. 2014. Inflammatory response caused by larvae and adults of Gnathostoma (Nematoda: Gnathostomatidae) in vertebrates of Mexico, including humans. Rev Mex Biodiv 85: 429-435. doi: 10.7550/ rmb.35496

16. Gill H, Paperna I. 2008. Proliferative visceral Isospora (atoxoplasmosis) with morbid impact on the Israeli sparrow Passer domesticus biblicus Hartert, 1904. Parasitol Res 103: 493-499. doi: 10.1007/s00436-008-0986-4 
17. Giraudeau M, Mousel M, Earl S, McGraw K. 2014. Parasites in the city: degree of urbanization predicts poxvirus and coccidian infections in house finches (Haemorhous mexicanus). PLoS One 9: e86747. doi : 10.1371/journal.pone. 0086747

18. Haustein T, Lawes M, Harris E, Chiodini PL. 2010. An eye-catching acanthocep-halan. Clin Microbiol Infect 16: 787-788. doi: 10.1111/j.14690691.2009.02896.x

19. Hilty S, Gwynne J, Tudor G, Meyer de Schaunsee R. 2003. Birds of Venezuela. $2^{\text {nd }}$ ed. USA: Princeton Univ Press. 878 p.

20. Kaufmann J. 1996. Parasites of poultry. In: Parasitic infections of domestic animals: a diagnostic manual. Germany: Basel Birkhäuser Verlag. p 337-393.

21. Minott P, Caballero M. 2007. Determinación de Salmonella spp y endoparásitos en zanates (Quiscalus mexicanus) del parque de Cañas, Guanacaste. Rev Costarric Salud Pública 16: 27-35.

22. Moore D. 1962. Morphology, life history, and development of the Acanthocephalan Mediorhynchus grandis Van Cleave, 1916. J Parasitol 48: 76-86. doi: 10.2307/ 3275416

23. Morales G, Pino de Morales L. 1977. Coprología. En: Manual de diagnóstico helmintológico en rumiantes. Venezuela: Colegio de Médicos Veterinarios del Estado Aragua. p 26-27.

24. Morand-Ferron J, Veillette M, Lefebvre L. 2006. Stealing of dunked food in carib grackles (Quiscalus lugubris). Behav Processes 73: 342347. doi: 10.1016/j.beproc.2006.08.006

25. Nakamura A, Meireles M. 2015. Cryptosporidium infections in birds - a review. Braz J Vet Parasitol 24: 253-267. doi: 10.1590/S1984-29612015063

26. Navarro-Lopez $R$, Vázquez-Mendoza, $L$, Villarreal C, Casaubon M, Marquez M. 2014. Highly pathogenic avian influenza $\mathrm{A} / \mathrm{H} 7 \mathrm{~N} 3$ in great-tailed grackles (Quiscalus mexicanus) in the Altos de Jalisco region of Mexico. 2014. JMM
Case Reports. [Internet]. Disponible en: http://jmmcr.sgmjournals.org/. doi: 10.1099/jmmcr.0.001461

27. Norton R, Ruff M. 2003. Internal parasites: nematodes and acanthocephalans. In: Saif Y, Barnes H, Fadly A, et $a l$. (eds.). Diseases of poultry. $11^{\text {th }}$ ed. Ames, Iowa: Blackwell Publishing. $p$ 931-960.

28. Phelps W Jr, Meyer de Schaunsee R. 1994. Una guía de las aves de Venezuela. $2^{\mathrm{a}}$ ed. Venezuela: Ed Ex Libris. $497 \mathrm{p}$.

29. Powell A, Barker K., Lanyon S. 2008. A complete species-level phylogeny of the grackles (Quiscalus spp.), including the extinct slender-billed grackle, inferred from mitocondrial DNA. The Condor 110: 718-728. doi: 10.1525/cond.2008.8633

30. Quah J, Ambu S, Lim Y, Mahdy M, Mak J. 2011. Molecular identification of Cryptosporidium parvum from avian hosts. Parasitology 138: 573-577. doi: 10.1017/S0031182010001691

31. Restrepo A, Carvajal H. 1982. Coccidias en aves silvestres. Actualidad Biol 11: 115-119.

32. Rodríguez-Ferraro A. 2015. Fishing behaviour of the carib grackle (Quiscalus lugubris) in Venezuela. Ornitol Neotrop 26: 207-209.

33. Rózsa L, Reiczigel J, Majoros G. 2000. Quantifying parasites in samples of hosts. J Parasitol 86: 228-232.

34. Ryan U, Hijjawi N. 2015. New developments in Cryptosporidium research. Int J Parasitol 45: 367-373. doi: 10.1016/j.ijpara.2015.01.009

35. Sanz V, Oviol L, Medina A, Moncada R. 2010. Avifauna del estado Nueva Esparta, Venezuela: recuento histórico y lista actual con nuevos registros de especies y reproducción. Interciencia 35 : 329-339.

36. Sevá A, Funada M, Richtzenhain L, Guimaraes M, Souza S, Allegretti L, Sinhorini J, et al. 2011. Genotyping of Cryptosporidium spp from free-living wild birds from Brazil. Vet Parasitol 175: 27-32. doi: 10.1016/j.vetpar.2010.09.031 
37. Shayegani M, Stone W, DeForge I, Root T, Parsons L, Maupin P. 1986. Yersinia enterocolitica and related species isolated from wildlife in New York State. Appl Environ Microbiol 52: 420-424.

38. Smales LR. 2002. Species of Mediorhynchus (Acanthocephala: Gigantorhynchidae) in Australia birds with the description of Mediorhynchus colluricinclae n. sp. J Parasitol 88: 375-
381. doi: 10.1645/0022-3395(2002)088[0375:SOMAGI]2.0.CO;2

39. Strewe R, Villa-De León C, Lobatón $G$ Morales A, Ayerbe F. 2006. Ampliación del rango de distribución del chango llanero Quiscalus lugubris (Icteridae) en Colombia. Rev Intrópica 3: 109-112.

40. Xiao L. 2010. Molecular epidemiology of Cryptosporidiosis: an update. Exp Parasitol 124: 80-89. doi: 10.1016/ j.exppara.2009.03.018 